ACTA MYCOLOGICA

Vol. 41 (2): 229-240

2006
Dedicated to Professor Alina Skirgietto

on the occasion of her ninety fifth birthday

\title{
Threatened and protected macromycetes in the Wkrzańska Forest
}

\author{
STEFAN FRIEDRICH \\ Department of Botany and Nature Protection, University of Agriculture \\ Słowackiego 17, PL 71434 Szczecin; sfriedrich@agro.ar.szczecin.pl
}

Friedrich S.: Threatened and protected macromycetes in the Wkrzańska Forest. Acta Mycol. 41(2): 229 240, 2006.

Eightysix interesting species of macromycetes, including fungi red listed in Poland (84 species) and fungi protected in Poland (11 species), observed in the Wkrzańska Forest are described. A total of 460 species of macromycetes were recorded during mycosociological studies conducted in the period between 2003 and 2005.

Key words: macromycetes, Wkrzańska Forest, threatened species, protected species, red list of fungi

\section{INTRODUCTION}

The Wkrzańska Forest is a vast complex of ca. $1550 \mathrm{~km}^{2}$ divided between Poland and Germany (Fig. 1). The Polish part, covering $340 \mathrm{~km}^{2}$ (Zarę ba 1986), comprises the Wkrzańska Plain and the northern part of the Szczecińskie Hills (Kondracki 1998). Leucobryo-Pinetum and Querco roboris-Pinetum phytocoenoses of various age dominate in the central and northern plain parts of the Wkrzańska Forest. Broadleaved and mixed tree-stands of the Luzulo pilosae-Fagetum and Fago-Quercetum petraeae associations occur in its western and southern parts on moraine hills. Small sections, scattered over the study area, are overgrown with the following associations: Ribeso nigri-Alnetum, Betulo pendulae-Quercetum roboris, Fraxino-Alnetum, Vaccinio uliginosiBetuletum pubescentis. The forests are administered by the Trzebież Forest District.

Macromycetes in the Wkrzańska Forest had not been examined before. Only 5 species of macromycetes common in Poland collected during studies on poplar mycotrophy have been reported from the area (Dominik, Ihnatowicz 1979): Laccaria laccata, Laetiporus sulphureus, Polyporus squamosus, Trametes versicolor and Vascellum pratense. On the other hand, the mycobiota of the adjacent regions has been investigated in relative depth: the Goleniowska Forest to the east of the study area (Friedrich 1984, 1985a, 1985b, 1997) and the city of Szczecin to the south (Friedrich 1987; Friedrich, Orzechowska 2002). Systematic mycological studies in the Wkrzańska Forest commenced in 2002. The occurrence and 


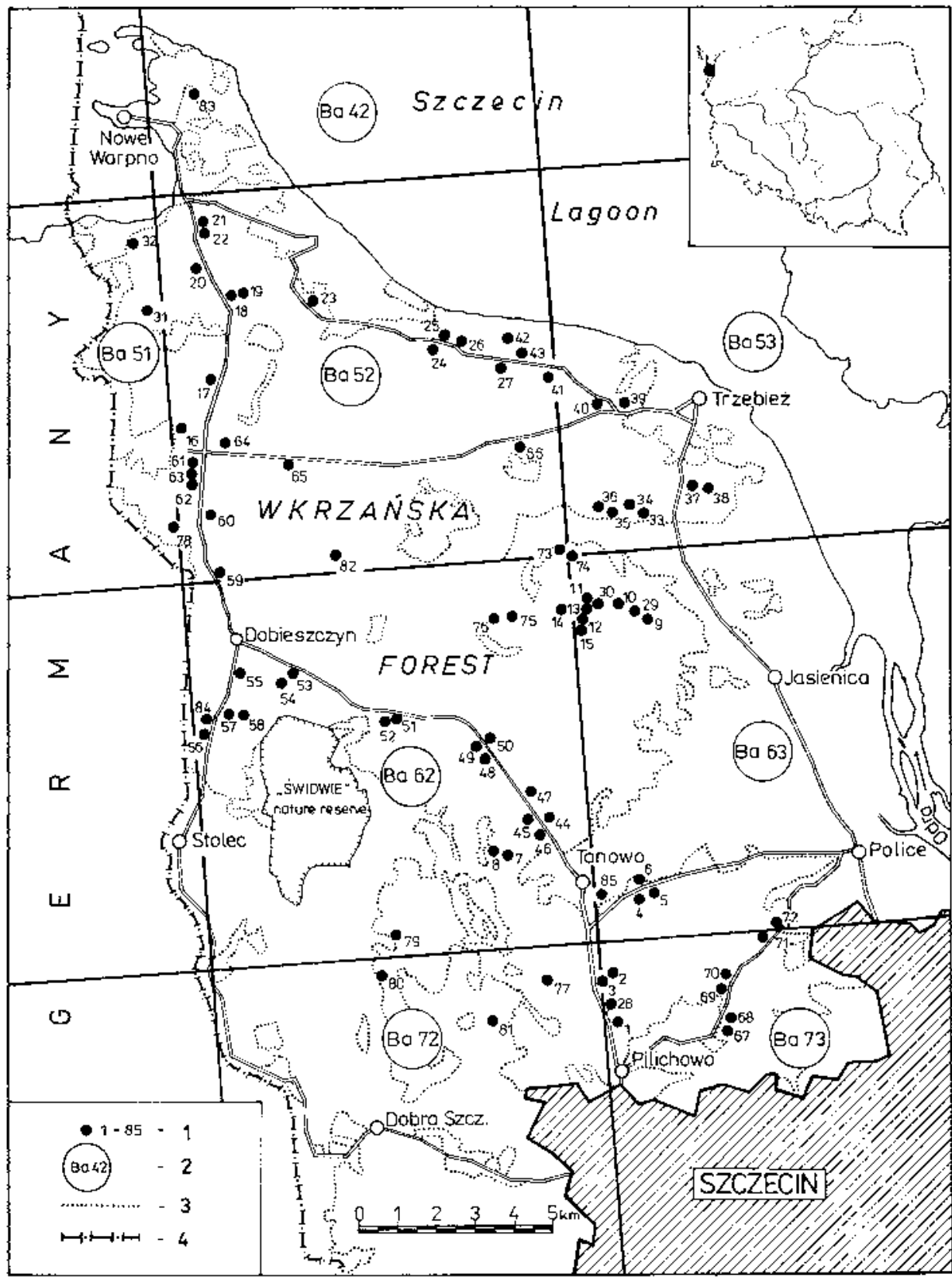

Fig. 1. Localization of macromycetes observation plots in Wkrzańska Primeval Forest: 1 observation plots (sites), 2 numbers of the grid ATPOL squares, 3 forests boundary, 4 state boundary. 
the distribution of threatened and protected macromycetes are presented below. The participation and the role of macromycetes in the forest communities of the Wkrzańska Forest will be discussed separately. Analysis results will be used to compile a regional list of threatened fungi in Pomerania.

\section{METHODS}

Systematic mycosociological research was conducted at 21 permanent observations plots, $400 \mathrm{~m}^{2}$ each, set up in forest communities. Community sociology was determined by the present author. Supplementary studies, that is individual mycological and phytosociological observations at additional 64 plots, also $400 \mathrm{~m}^{2}$ each, were carried out throughout the periods of increased fungal fruiting. The sites were situated in the ATPOL grid squares (Tab. 1). Fruiting abundance was evaluated in the Moser's scale (1949). The growth manner of the fruit-bodies on the substrate was also identified. Mycological observations were additionally performed outside the forest areas (roadsides, forest tracts, glades, etc.) using the itinerary method, fungal species were recorded, and occurrence conditions were noted. Studies were continued over 3-4 years in permanent plots which were examined 5 times per year on average.

Fungal taxa of red-listed threatened species (Wojewoda, Ławrynowicz 1992) and protected species (Dz. U. 2004.168.1765) were included in the study.

\section{RESULTS AND DISCUSSION}

The occurrence of 460 fungal species belonging to all ecological groups were recorded during studies conducted at 85 sites between 2002 and 2005. A high percentage of mycorrhizal fungi which constitute $38 \%$ of the recorded macromycetes should be stressed. The participation of other groups is as follows: lignicolous fungi - over $30 \%$, litter-inhabiting and bryophilous fungi - over $23 \%$, humicolous saprobes - over $6 \%$, plant and fungal parasites - less than 3\%. Many of these taxa are threatened or rare not only in Poland.

The occurrence of 86 interesting species collected between 2002 and 2005 is described below. A total of 84 red-listed species threatened in Poland and 11 species protected in Poland were recorded in the mycobiota of the Wkrzańska Forest. One species is classified as extinct (Ex), 4 species are classified as endangered (E), 16 species are classified as vulnerable (V), 31 species are classified as rare (R), and 32 species are classified as indeterminate (I). Some threatened species are protected, and only 2 protected species (Langermannia gigantea, Meripilus giganteus) are not red-listed.

The nomenclature and systematics of the orders follow Kirk et al. (2001), and the nomenclature of the species follows many sources cited by Wojew od a (2003), mostly: Antonín \& Noordeloos (1997), Hansen \& Knudsen (1992, 1997, 2000), Kreisel (1987), Krieglsteiner G. J. (1991), Krieglsteiner L. (1999). Species within orders are reported alphabetically.

Threat category and protection category as well as the substrate type are given for each species, and the plant community in which the species occurs is specified. The following data are also provided: quantity range of the occurrence of fruit-bod- 
Table 1

Sites of macromycetes in the Wkrzańska Forest

\begin{tabular}{|c|c|c|c|c|c|c|c|c|c|}
\hline $\mathrm{N}^{\mathrm{o}}$ & ATPOL & Forest district & $\begin{array}{l}\text { Forest } \\
\text { division }\end{array}$ & $\begin{array}{c}\text { Plant } \\
\text { association }\end{array}$ & $\mathrm{N}^{\circ}$ & ATPOL & Forest district & $\begin{array}{c}\text { Forest } \\
\text { division }\end{array}$ & $\begin{array}{c}\text { Plant } \\
\text { association }\end{array}$ \\
\hline 1 & Ba73 & Tanowo & $813 c$ & $L P$ & 39 & Ba53 & Pienice & $110 \mathrm{i}$ & $Q r P$ \\
\hline $2 *$ & Ba73 & Tanowo & $771 \mathrm{~g}$ & $L P$ & 40 & Ba53 & Pienice & $148 b$ & $\begin{array}{l}\text { pine } \\
\text { greenwood }\end{array}$ \\
\hline 3 & Ba72 & Tanowo & 772 & $F Q p$ & 41 & Ba52 & Pienice & $115 \mathrm{a}$ & $L P$ \\
\hline $4^{*}$ & Ba63 & Tanowo & $718 \mathrm{~g}$ & $L P$ & 42 & Ba52 & Pienice & $83 \mathrm{~g}$ & $L p F$ \\
\hline 5 & Ba63 & Tanowo & $718 \mathrm{f}$ & $Q r P$ & 43 & Ba52 & Pienice & 821 & $Q r P$ \\
\hline 6 & Ba63 & Tanowo & $718 \mathrm{~b}$ & $\begin{array}{l}\text { pine } \\
\text { greenwood }\end{array}$ & 44 & Ba62 & Tatynia & $666 \mathrm{~d}$ & $L p F$ \\
\hline 7 & Ba62 & Tanowo & $669 \mathrm{~d}$ & $L P$ & $45^{*}$ & Ba62 & Tatynia & $667 b$ & $L p F$ \\
\hline 8 & Ba62 & Tanowo & $670 \mathrm{j}$ & $\begin{array}{l}\text { pine } \\
\text { greenwood }\end{array}$ & 50 & Ba62 & Tatynia & $577 \mathrm{~g}$ & $L P$ \\
\hline 9 & Ba63 & Tusznica & $589 \mathrm{a}$ & $L P$ & 51 & Ba62 & Tatynia & $531 \mathrm{c}$ & $Q r P$ \\
\hline 10 & Ba63 & Tusznica & $564 \mathrm{~b}$ & $L P$ & 52 & Ba62 & Zalesie & $532 \mathrm{~b}$ & $Q r P$ \\
\hline 11 & Ba63 & Tusznica & 5161 & $L p F$ & 53 & Ba62 & Podymin & $440 \mathrm{~h}$ & $L P$ \\
\hline 12 & Ba63 & Tusznica & $542 \mathrm{f}$ & $L p F$ & $55^{*}$ & Ba62 & Podymin & $444 f$ & $L p F$ \\
\hline 14 & Ba62 & Tusznica & $518 \mathrm{~g}$ & $L P$ & 58 & Ba62 & Zalesie & $490 \mathrm{a}$ & $L p F$ \\
\hline 16 & Ba52 & Myślibórz & $260 \mathrm{~d}$ & $L p F$ & 59 & Ba52 & Podymin & $384 \mathrm{f}$ & $L P$ \\
\hline 17 & Ba52 & Myślibórz & $174 \mathrm{f}$ & $Q r P$ & 63 & Ba52 & Myślibórz & $299 \mathrm{i}$ & $L p F$ \\
\hline $18^{*}$ & Ba52 & Nowe Warpno & $66 \mathrm{j}$ & $L P$ & 64 & Ba52 & Myślibórz & $257 \mathrm{~b}$ & $L P$ \\
\hline 19 & Ba52 & Nowe Warpno & $66 \mathrm{~h}$ & $L P$ & 65 & Ba52 & Myślibórz & $293 b$ & $L P$ \\
\hline 20 & Ba52 & Nowe Warpno & $39 \mathrm{~g}$ & $\begin{array}{l}\text { pine } \\
\text { greenwood }\end{array}$ & 66 & Ba52 & Mazańczyce & $197 \mathrm{c}$ & $L P$ \\
\hline 21 & Ba52 & Nowe Warpno & $23 \mathrm{~d}$ & $L P$ & $67^{*}$ & Ba73 & Siedlice & $827 \mathrm{c}$ & $L p F$ \\
\hline 22 & Ba52 & Nowe Warpno & $23 \mathrm{f}$ & $Q r P$ & $68^{*}$ & Ba73 & Siedlice & $826 \mathrm{~d}$ & $F Q p$ \\
\hline 23 & Ba52 & Nowe Warpno & $61 \mathrm{ab}$ & $B p Q r$ & 69 & $\mathrm{Ba} 73$ & Siedlice & $807 \mathrm{j}$ & $L p F$ \\
\hline $24^{*}$ & Ba52 & Pienice & $89 d$ & $L p F$ & 71 & Ba73 & Siedlice & $801 \mathrm{~g}$ & $Q r P$ \\
\hline 25 & Ba52 & Pienice & $89 \mathrm{c}$ & $F Q p$ & $72 *$ & Ba63 & Siedlice & $801 \mathrm{c}$ & $F Q p$ \\
\hline $26 *$ & Ba52 & Pienice & $86 \mathrm{~h}$ & $F Q p$ & 77 & Ba72 & Tanowo & $751 \mathrm{~m}$ & $L P$ \\
\hline 27 & Ba52 & Pienice & $119 \mathrm{~d}$ & $L p F$ & 78 & Ba51 & Podymin & $342 d$ & $L p F$ \\
\hline 28 & Ba73 & Tanowo & $792 n$ & $Q r P$ & 79 & Ba62 & Dobra & $693 d$ & $L p F$ \\
\hline 29 & Ba63 & Tusznica & $565 \mathrm{a}$ & $Q r P$ & 81 & Ba72 & Tanowo & $797 \mathrm{a}$ & $L P$ \\
\hline $30 *$ & Ba63 & Tusznica & $541 \mathrm{c}$ & $R n A$ & 82 & Ba52 & Drogoradz & $360 \mathrm{c}$ & raised bog \\
\hline 35 & Ba53 & Mazańczyce & $270 \mathrm{~g}$ & $L p F$ & 83 & Ba42 & Nowe Warpno & $4 d$ & $B p Q r$ \\
\hline $36^{*}$ & Ba53 & Mazańczyce & $271 d$ & $B p Q r$ & 84 & Ba62 & Zalesie & $491 d$ & $\begin{array}{l}\text { pine } \\
\text { greenwood }\end{array}$ \\
\hline $37 *$ & Ba53 & Mazańczyce & $226 \mathrm{~d}$ & $Q r P$ & 85 & Ba63 & Tanowo & $720 \mathrm{~b}$ & $\begin{array}{l}\text { pine } \\
\text { greenwood }\end{array}$ \\
\hline $38^{*}$ & Ba53 & Mazańczyce & $226 \mathrm{~b}$ & $R n A$ & & & & & \\
\hline
\end{tabular}


ies in the Mos e r's scale (1949), record date and a list of localities recorded between 2002 and 2005 preceded by their number in parentheses. The quantity is not given for species producing permanent fruit-bodies. The Latin plant nomenclature follows Mirek et al. (2002) and that of communities follows Matuszkiewicz (2001).

\section{Abbreviations:}

Threat categories in Poland (Wojewoda, Ławrynowicz 1992): PL Ex extinct, PL E endangered, PL V vulnerable, PL R rare, PL I indeterminate; $\S$ fully protected species, $\S$ partially protected species (Regulation of the Minister of the Environment of $9^{\text {th }}$ July 2004); RnA Ribeso nigri Alnetum, LP Leucobryo Pinetum, QrP Querco roboris Pinetum, BpQr Betulo pendulae Quercetum roboris, FQp

Fago Quercetum petraeae, $L p F \quad$ Luzulo pilosae Fagetum; + 5 quantity range of fruit bodies according to Moser (1949); Jan. January, Aug. August, Sept. September, Oct. October, Nov. November, Dec. December; * permanent observation plot.

\section{ASCOMYCETES}

\section{Helotiales}

Ascotremella faginea (Peck) Seaver - PL-V; on a fallen branch of Fagus sylvatica; LpF; 1; July 2004; site (1): 69, (Fig. 2).

Bulgaria inquinans (Pers.: Fr.) Fr. - PL-R; on logs and fallen branches of Quercus petraea and Q. robur; BpQr, QrP; 3; Oct. 2004, Nov. 2005; sites (2): 36*, 51.

Chlorencoelia versiformis (Pers.: Fr.) Dixon - PL-I; on a log of Quercus robur; BpQr; 1; Oct. 2004; site (1): 83.

\section{BASIDIOMYCETES Dacrymycetales}

Calocera furcata (Batsch.: Fr.) Fr. - PL-I; on logs and fallen branches of Pinus sylvestris; LP, QrP; 2-4; July-Nov. 2002-2005; sites (12): 7, 8, 9, 12, 19, 39, 40, 43, 53, $69,84,85$.

\section{Tremellales}

Tremella foliacea Pers. - PL-I; on trunks and logs of Betula pendula; LP; +-1; July 2004, Oct. 2005; sites (3): 4*, 14, 64.

Tremella globospora D. A. Reid - PL-I; on old stromata of pyrenomycetous fungi growing on woods; pine greenwood; 2; Oct. 2002; site (1): 8, (Fig. 3).

\section{Agaricales}

Amanita citrina var. alba (Gillet) Gilbert - PL-I; on the ground under Pinus sylvestris; LP; +-1; Oct. 2005; sites (2): 2*, 65.

Amanita virosa (Fr.) Bertillon - PL-V; on the ground under Pinus sylvestris; LP; 1 ;

Sept. 2003; site (1): 2*. 
Arrhenia retiruga (Bull.: Fr.) Redhead - PL-I; on Mnium hornum moss; RnA: 2.2; Nov. 2004; site (1): 38*, (Fig. 4).

Arrhenia spathulata (Fr.: Fr.) Redhead - PL-I; on Tortula ruralis moss; roadside; Aug. 2004; site (1): near Karszno.

Clitocybe candicans (Pers.: Fr.) P. Kumm. - PL-I; on the litter; RnA, QrP, FQp, LpF; 2.2; Sept.-Nov. 2003-2005; sites (4): 3, 24*, 29, 38*.

Clitocybe hydrogramma (Bull.: Fr.) P. Kumm. - PL-R; on the broadleaved litter; LpF; Nov. 2004; site (1): 16.

Coprinus picaceus (Bull.: Fr.) Gray - PL-V; on the ground; LpF; +-1; Sept.-Oct. 2004, 2005; sites (2): 16, $67^{*}$.

Cortinarius armeniacus (Schaeff.: Fr.) Fr. - PL-I; on the ground; LP; 1; Sept. 2004; sites (2): 41, 81.

Cortinarius orellanus Fr. - PL-I; on the ground; QrP; 1-2; July 2004; site (1): 17.

Cystodermella granulosum (Batsch: Fr.) Harmaja - PL-R; on the ground; QrP, +-1; Sept.-Oct. 2004; sites (2): 17, 28.

Entoloma juncinum (Kühner. \& Romagn.) Noordel. - PL-I; on the litter; FQp; 1 ; July 2004; site (1): 25.

Entoloma papillatum (Bres.) Dennis - PL-I; on the ground; QrP; 1; Oct. 2005; site (1): 51.

Fayodia maura (Fr.) Singer - PL-I; on a charred branch of Pinus sylvestris; LP; 1; Oct. 2002; site (1): near 85.

Fistulina hepatica (Schaeff.): Fr. - PL-V, §§; on a trunk of Quercus robur; 1; Sept. 2005; site (1): near 45*.

Galerina paludosa (Fr.) Kühn. - PL-I; among Sphagnum spp.; raised bog on Lake Piaszynko; 2; July 2004; site (1): 82.

Galerina sphagnorum (Pers.: Fr) Kühner - PL-I; among Sphagnum spp.; raised bog on Lake Piaszynko; 2; July 2004; site (1): 82.

Galerina triscopa (Fr.) Kühner - PL-I; on a log of Fagus sylvatica; LpF; 1; Oct. 2004; site (1): 27.

Gymnopus putillus (Fr.: Fr.) Antonín, Halling \& Noordel. - PL-I; on the litter; QrP; 1; Aug. 2004, Sept. 2005; site (1): 22.

Inocybe acuta Boud. - PL-R; on the ground; QrP; 1; July 2004; site (1): 43.

Inocybe calospora Quél. - PL-R; on the ground; RnA; July 2004; site (1): 30*.

Langermannia gigantea (Batsch: Pers.) Rostk. - $\S \S$; on the ground; July 2004, Sept. 2005; site (1): the Świdwie Ornithological Station, (Fig. 5).

Lepiota tomentella J. E. Lange - PL-I; on the ground; LP; 1; Oct. 2003; site (1): 66.

Lyophyllum palustris (Peck) Singer - PL-I; among Sphagnum spp.; raised bog on Lake Piaszynko; 2; Sept. 2004; site (1): 82.

Macrolepiota procera (Scop.: Fr.) Singer - PL-I; on the ground; $Q r P, L p F$, pine greenwood, roadside; +-1; Sept.-Nov. 2002-2005; sites (5): 5, near 6, 20, 51, 64.

Macrolepiota rhacodes (Vittad.) Singer - PL-I; on the ground; QrP, forest roadside; 1; July 2004; sites (2): near 28, 71.

Marasmiellus foetidus (Sowerby: Fr.) Antonín, Halling \& Noordel. - PL-R; on fallen twigs of Fagus sylvatica; LpF; 2; Nov. 2005; site (1): 42.

Mycena adonis (Bull.: Fr.) Gray - PL-R; on the litter and on fallen twigs of Pinus sylvestris; LP; 2; Oct. 2004; site (1): 81. 
Mycena atroalba (Bolt.: Fr.) Gillet - PL-R; on a log of Pinus sylvestris, 2; Sept. 2003; site (1): 28.

Mycena belliae (Johnst.) P. D. Orton - PL-R; on culms of Phragmites australis; Phragmitetum australis; 2-3; Sept.-Nov. 1999-2004 (leg. K. Olszanowski); site (1): Lake Świdwie.

Mycena crocata (Schrad.: Fr.) P. Kumm. - PL-R; on fallen twigs of Fagus sylvatica; LpF; 2; Nov. 2005; sites (2): 58, 67*.

Mycena olivaceomarginata (Massee) Massee - PL-R; on the coniferous litter; QrP; 2; Sept. 2004; site (1): 71.

Mycena pelianthina (Fr.) Quél. - PL-I; on the broadleaved litter; LpF; 1-2; July 2004, Nov. 2005; sites (3): 24*, 45*, 63.

Mycena purpureofusca (Peck) Sacc. - PL-V; on a stump of Pinus sylvestris; LpF; 2; Oct. 2004; site (1): $45^{*}$.

Mycena septentrionalis Maas Geest. - PL-R; on the litter; LP; 1; Sept. 2004; site (1): 59.

Mycena viridimarginata P. Karst. - PL-V; on a stump of Fagus sylvatica; LpF; July 2004; site (1): 44.

Omphaliaster asterosporus (J. E. Lange) Lamoure - PL-E; on the litter among mosses; LP; 1-2; Nov. 2005; sites (3): 7, 9, 64.

Oudemansiella mucida (Schrad.: Fr.) Höhn. - PL-V; on trunks, logs and fallen branches of Fagus sylvatica; LpF; 2-3; July-Nov. 2003-2005; sites (6): 24*, 35, 45*, $55^{*}, 67^{*}, 79$, (Fig. 6).

Pleurotus dryinus (Pers.: Fr.) P. Kumm. - PL-R; on stumps of Alnus glutinosa; RnA; 1; Nov. 2003; site (1): $30^{*}$.

Pluteus atromarginatus (Singer) Kühner - PL-V; on stumps of Pinus sylvestris; LP; +1; July 2004, Sept.-Oct. 2005; sites (3): 37*, 50, 64.

Psathyrella gracilis (Fr.: Fr.) Quél. - PL-I; on the ground; LpF; 1; Oct. 2003; site (1): 35.

Psilocybe elongata (Pers.: Fr.) J. E. Lange - PL-V; among Sphagnum spp.; raised bog on Lake Piaszynko; 1; Oct. 2004; site (1): 82.

Psilocybe polytrichi (Fr.: Fr.) Pears. \& Dennis - PL-R; on the litter among mosses; LP; Oct. 2002, Nov. 2004, Sept. 2005; sites (4): 14, 19, 64, 77.

Psilocybe uda (Pers.: Fr.) Gillet - PL-R; among Sphagnum spp.; raised bog on Lake Piaszynko; 2; Oct. 2005; site (1): 82.

Rhodocollybia prolixa (Hornem.: Fr.) Antonín \& Noordel. var. distorta (Fr.) Antonín, Halling et Noordel. - PL-I; on the litter; LP, QrP; 1-2; Sept. 2004, Oct. 2005; sites (2): 21, 29.

Tricholoma equestre (L.: Fr.) P. Kumm. ss. lato - PL-I; on the ground under Pinus sylvestris; LP, pine greenwood; 1-2; Oct. 2003, Nov. 2005; sites (2): 2*, 66.

Tricholoma pessundatum (Fr.: Fr.) Quél. - PL-I; on the ground under Pinus sylvestris; LP; 1; Sept. 2004; site (1): 77.

Tricholoma sejunctum (Sowerby: Fr.) Quél. - PL-I; on the ground under Fagus sylvatica and Quercus petraea; FQp; 1; Oct. 2005; site (1): 54. 


\section{Boletales}

Boletus edulis Bull.: Fr. - PL-V; on the ground under: Fagus sylvatica, Pinus sylvestris, Quercus robur; QrP, LpF, July, Oct. 2004, 2005; +-1; sites (4): 5, 51, 55*, 58.

Boletus pinophilus Pilát \& Dermek - PL-I; on the ground under Pinus sylvestris; LP; +-1; July 2004, Sept. 2005; site (1): 18*.

Boletus suspectus Krombh. - PL-E; on the ground under Fagus sylvatica; LpF; 1; Nov. 2005; site (1): 24*.

Gomphidius roseus (Fr.) Fr. - PL-R; on the ground; LP; +: Oct. 2004, 2005; sites (2): 7, 64, (Fig. 7).

Gyrodon lividus (Bull.: Fr.) Sacc. - PL-R; on the ground; RnA; 1; July 2004; site (1): $38^{*}$, (Fig. 8).

\section{Cantharellales}

Cantharellus cibarius Fr. - PL-I; on the ground under Pinus sylvestris; LP; 1-2; JulyOct. 2003-2005; sites (5): 2*, 19, 41, 66, 77.

Clavulina rugosa (Bull.: Fr.) J. Schröt. - PL-R; on the ground; LP, pine greenwood; 1-2; Sept. 2004; sites (2): 8, 19.

\section{Hymenochaetales}

Inonotus obliquus (Pers.: Fr.) Pilát - PL-R, §; on a trunk of Betula pendula; LP; Jan.Dec. 2004-2005; sites (2): 10, 18 *.

\section{Phallales}

Clavariadelphus fistulosus (Holmsk.: Fr.) Corner - PL-R; on fallen twigs of Betula pendula; LP; 1-2; Oct. 2002; sites (2): 2*, 4*, (Fig. 9).

Geastrum fimbriatum Fr. - PL-R, §§; on the ground; FQp; 2; July 2004; site (1): 68*. Geastrum rufescens Pers.: Pers. - PL-E, §§; on the ground; FQp; 1; Oct. 2003; site (1): $26^{*}$.

Phallus duplicatus Bosc - PL-E; on the ground; LpF; +; July 2004; site (1): 55*.

\section{Polyporales}

Datronia mollis (Sommerf.: Fr.) Donk - PL-I; on stumps and logs of Fagus sylvatica; LpF; Jan.-Dec. 2004-2005; sites (3): 11, 16, 45*.

Ganoderma lucidum (M. A. Curtis: Fr.) P. Karst. - PL-R, §§; on a stump of Fagus sylvatica; LpF; Sept. 2004; site (1): 24*.

Ischnoderma benzoinum (Wahlenb.: Fr.) P. Karst. - PL-R; on a log of Pinus sylvestris; $L P$; 1; Sept. 2003, Oct. 2005; site (1): 2*.

Ischnoderma resinosum (Fr.) P. Karst. - PL-V; on a log of Fagus sylvatica; LpF; 2; Oct. 2005; site (1): 45*, (Fig. 10). 
Lentinus tigrinus (Bull.: Fr.) Fr. - PL-I; on a log of Alnus glutinosa and on a fallen branch of Betula pendula; RnA, BpQr; 1-2; Sept. 2003, Oct. 2005; sites (2): 23, $38^{*}$.

Meripilus giganteus (Pers.: Fr.) P. Karst. - $\S$; at the bases of stumps and living trunks of Fagus sylvatica; LpF, FQp; July-Aug. 2004, 2005; sites (7): 35, 45* , 58, 63, 67*, 72*, 78, (Fig. 11).

Oligoporus ptychogaster (Ludwig) R. \& O. Falck - PL-R; on a log and on the coniferous litter of Pinus sylvestris; LP; 2; Sept. 2004; sites (2): 2*, 64, (Fig. 12).

Phaeolus schweinitzii (Fr.: Fr.) Pat. - PL-R; on trunks of Pinus sylvestris; LP, QrP; Nov. 2004; sites (2): 21, 37*.

Pycnoporus cinnabarinus (Jacq.: Fr.) P. Karst. - PL-R; on logs and fallen branches of Betula pendula and Fagus sylvatica; LP, FQp; Jan.-Dec. 2002-2004; sites (4): 25, 41, 50, 66, (Fig. 13).

Sparassis brevipes Krombh. - PL-V, §§; on a fallen branch of Fagus sylvatica; LpF; +; Nov. 2005; site (1): 24*, (Fig. 14).

Sparassis crispa (Wulf.): Fr. - PL-R, §§; on roots of Pinus sylvestris; LP, QrP; +-1; Sept.-Oct. 2003-2005; sites (6): 1, 9, 22, 37*, 51, 77, 83.

\section{Russulales}

Hericium coralloides (Scop.: Fr.) Pers. - PL-V, §§; on logs of Fagus sylvatica; LpF; +1; Sept.- Oct. 2005; sites (3): 16, 67*, 79 (Fig. 15).

Lactarius chrysorrheus Fr. - PL-R; on the ground under Quercus petraea and Q. robur; FQp, QrP; 1-2; Sept.-Oct. 2004, Oct. 2005; sites (3): 25, 52, 68 *.

Lactarius deliciosus (L.: Fr.) Gray - PL-V; on the ground under Pinus sylvestris; LP; 1-2; Oct. 2004, Sept. 2005; site (1): 21.

Russula alutacea (Pers.: Fr.) Fr. - PL-I; on the ground under Fagus sylvatica; LpF, QrP; +-1; Oct. 2003, Sept. 2004; sites (3): 11, 52, 69.

Russula coerulea (Pers.) Fr. - PL-Ex; on the ground under Pinus sylvestris; LP; 1; Oct. 2004; site (1): 65.

Russula olivacea (Schaeff.) Fr. - PL-R; on the ground under Fagus sylvatica; LpF, FQp; 1-2; Oct. 2003, Sept. 2004, Oct. 2005; sites (3): 27, 72*, 79.

Stereum subtomenosum Pouzar - PL-R; on logs of Quercus petraea and Fagus sylvatica; LpF, FQp; Jan.-Dec. 2004-2005; sites (3): 35, 44, 68*.

\section{Thelephorales}

Phellodon tomentosus (L.: Fr.) Banker - PL-V; on the ground in the litter; LP; 1; Oct. 2003; site (1): 19.

Sarcodon imbricatus (L.: Fr.) P. Karst. - PL-V, §§; on the ground; LP; 1; Oct. 2004; site (1): $2^{*}$.

Thelephora caryophyllea (Schaeff.): Fr. - PL-R; on the ground; LP; 1; Sept. 2004; site (1): 19. 


\section{CONCLUSIONS}

As many as 50 species of the threatened and protected taxa are very rare and occur only at 1 locality in the Wkrzańska Forest. Twenty-six species were recorded at 2-3 localities, 6 species - at 4-5 localities, and 3 species - at 6-7 localities (Sparassis crispa, Oudemansiella mucida, Meripilus giganteus). Among the taxa studied, Calocera furcata, recorded at 12 localities, is the most common one in the Wkrzańska Forest.

Species previously not reported from Pomerania occur in the Wkrzańska Forest. These are for instance: Arrhenia retiruga, A. spathulata, Ascotremella faginea, Ischnoderma benzoinum, I. resinosum, Omphaliaster asterosporus, Phallus duplicatus, Sparassis brevipes, Tremella globospora. Species very rare in Poland, including taxa previously known from 1-3 localities in Poland, occur in the studied mycobiota. These are: Phallus duplicatus, known only from 1 site, Arrhenia retiruga, known from 2 localities, and Ascotremella faginea, Russula coerulea and Tremella globospora, known from 3 localities. It should be stressed that the two former species were previously recorded 70-90 years ago.

It should be noticed that many species of macromycetes rare in Poland, occurring at a few localities, are frequent or even common in Germany, especially in its southern part (Wojewoda 2003; Kreisel 1987; Krieglsteiner G. J. 1991). Phallus duplicatus, whose locality in the Wkrzańska Forest is the second site in Poland, is the best example. The taxon occurs at ca. 50 localities in Germany, including the Lands adjacent to the study area (Meklenburg - Western Pomerania, Brandenburg). Other species in this group include: Boletus suspectus, Entoloma papillatum, Inocybe acuta, Ischnoderma resinosum, Marasmiellus foetidus, Russula coerulea and Sparassis brevipes.

As Russula coerulea is recorded at the fourth locality in Poland, the species should be reclassified from extinct (Ex) to endangered (E), as suggested by Wojewoda (2003).

Over $52 \%$ threatened and protected species were recorded only in sporadic plots and during itinerary observations while only nearly $20 \%$ were recorded exclusively in permanent plots. The results confirm the significance of observations conducted outside permanent plots for the investigation of the biodiversity of macromycetes in the study area.

\section{REFERENCES}

Antonín V., Noordeloos E. 1997. A Monograph of Marasmius, Collybia and related genera in Europe. Part 2: Collybia, Gymnopus, Rhodocollybia, Crinipellis, Chaetocalathus, and additions to Marasmiellus. Libri Bot. 17. IHW Verlag, München.

Dominik T., Ihnatowicz A. 1979. Badania mikotrofizmu topól w uprawach doświadczalnych Insty tutu Badawczego Leśnictwa na terenie woj. szczecińskiego. Prace IBL. 544: 67112.

Dz.U.2004.168.1765. Rozporządzenie Ministra Środowiska z dnia 9 lipca 2004 r. w sprawie gatunków dziko występujących grzybów objętych ochroną.

Friedrich S. 1984. Mikoflora Puszczy Goleniowskiej. Acta Mycol. 20 (2): 173208.

Friedrich S. 1985 a. Macromycetes na tle zespołów leśnych Puszczy Goleniowskiej. Acta Mycol. 21 (1): 4376.

Friedrich S. 1985 b (1987). Charakterystyka ekologiczno fenologiczna macromycetes Puszczy Gole niowskiej. Acta Mycol. 21 (2): 143164.

Friedrich S. 1987. Macromycetes Szczecina. Bad. Fizjogr. nad Pol. Zach. 38, Ser. B. 526. 
Friedrich S. 1997. Macromycetes of the proposed nature reserve Wilcze Uroczysko Olszanka in the Odra estuary. Acta Mycol. 32: 239255.

Friedrich S., 2004. Wstępne wyniki badań macromycetes Puszczy Wkrzańskiej. (In:) E. Jendrzej czak (ed.). Przyroda Polski w europejskim dziedzictwie dóbr natury. Materiały 53 Zjazdu PTB Toruń Bydgoszcz: 133134.

Friedrich S., Orzechowska M. 2002. Macromycetes w środowisku miejskim Szczecina. Bad. Fizjogr. nad Pol. Zach. Ser. B. 51: 730.

Hansen L., Knudsen H. 1992 (eds). Nordic Macromycetes. 2. Polyporales, Boletales, Agaricales, Russulales. Nordsvamp, Copenhagen.

Hansen L., Knudsen H. 1997. (eds). Nordic Macromycetes. 3. Heterobasidioid, Aphyllophoroid and Gasteromycetoid. Basidiomycetes. Nordsvamp, Copenhagen.

Hansen L., Knudsen H. 2000. (eds). Nordic Macromycetes. 1. Ascomycetes. Nordsvamp, Copenha gen.

Kirk M. P., Cannon P. F., David J. C., Stalpers J. A. 2001. Ainsworth \& Bisby's Dictionary of the Fungi. Ed. 9th. CAB International, Wallingford.

Kondracki J. 1998. Geografia regionalna Polski. PWN, Warszawa.

Kre isel H. 1987. Pilzflora der Deutschen Demokratischen Republik. Basidiomycetes (Gallert, Hut und Bauchpilze). VEB Gustav Fischer Verlag Jena.

Krieglsteiner G. J. 1991. Verbreitungsatlas der Großpilze Deutschlands (West). Band 1: Ständer pilze. Teil A: Nichtblätterpilze, Teil B: Blätterpilze. Verlag Eugen Ulmer, Stuttgart.

Krieglsteiner L. 1999. Pilze im Naturraum Mainfränkische Platten und ihre Einbindung in die Ve getation. Regensb. Mycol. Schr. 9: 1906.

Matuszkiewicz W. 2001. Przewodnik do oznaczania zbiorowisk roślinnych Polski. PWN, Warszawa.

Mirek Z., Piękoś Mirkowa H., Zając A., Zając M.2002. Flowering plants and pterido phytes of Poland a checklist. (In:) Z. Mire k (ed.). Biodiversity of Poland. 1. W. Szafer Institute of Botany, Polish Academy of Sciences, Kraków.

Moser M. 1949. Untersuchungen über den Einfluss von Waldbränden auf die Pilzvegetation I. Sydo wia, Ann. Mycol. 3 (1/6): 336383.

Wojewoda W., Lawrynowicz M. 1992. Red list of threatened macrofungi in Poland. (In:) K. Za rzycki, W. Wojewoda, Z. Heinrich (eds). List of threatened plants in Poland. 2 ed.: 2756. W. Szafer Institute of Botany, Polish Academy of Sciences, Kraków.

Wojewoda W. 2003. Checklist of Polish larger Basidiomycetes. (In:) Z. Mirek (ed.). Biodiversity of Poland. 7. W. Szafer Institute of Botany, Polish Academy of Sciences, Kraków.

Z a rę b a R. 1986. Puszcze, bory i lasy Polski. PWRiL, Warszawa.

\section{Zagrożone i chronione macromycetes Puszczy Wkrzańskiej}

\section{Streszczenie}

W pracy przedstawiono charakterystykę występowania wybranych taksonów grzybów wiel koowocnikowych Puszczy Wkrzańskiej. Jest to część wyników systematycznych badań miko socjologicznych prowadzonych na 21 stałych i 64 sporadycznych powierzchniach obserwacyj nych, w latach 2002 2005. W wyniku tych badań stwierdzono 460 gatunków macromycetes, spośród których w niniejszej pracy zaprezentowano 86 gatunków. Są to taksony znajdujące się na Czerwonej Liście grzybów zagrożonych w Polsce (84 gatunki) oraz grzyby chronione w Polsce (11 gatunków). Charakterystyka poszczególnych gatunków obejmuje: kategorię za grożenia i formę ochrony, podłoże, na którym rozwijały się owocniki, zbiorowisko roślinne, ilościowość wytwarzania owocników, okres występowania owocników oraz liczbę i wykaz sta nowisk. 
